\title{
Nonlinear interaction of copropagating and counterpropagating waves in straight and highly twisted single-mode fibers
}

\author{
B. Crosignani* and A. Yariv \\ California Institute of Technology, Pasadena, California 91125
}

Received August 10, 1987; accepted November 2, 1987

\begin{abstract}
We derive the system of equations describing the nonlinear interaction, associated with the optical Kerr effect, among the four forward- and backward-propagating modes in a straight single-mode fiber. This allows us, in particular, to obtain the set of equations governing nonlinear evolution in a highly twisted fiber of the corresponding copropagating and counterpropagating right and left circularly polarized modes.
\end{abstract}

\section{INTRODUCTION}

There has been recently a great deal of interest in nonlinear propagation in single-mode optical fibers. In particular, the propagation effects associated with the presence of a nonlinear contribution to the refractive index $n$ proportional to the instantaneous intensity of the field $E\left(n=n_{1}+n_{2}|E|^{2}\right.$, optical Kerr effect) may become important at relatively low optical powers over the diffraction-free interaction length provided by the fiber. In general, there exist two regimes of propagation, according to whether chromatic dispersion can be assumed to be negligible or not. While the second situation (large fiber lengths) concerns mainly telecommunications since the interplay between nonlinearity and chromatic dispersion can lead to a substantial reshaping of a propagating optical pulse (e.g., envelope solitons ${ }^{1}$ ), the first situation (short fiber lengths) is more relevant to all-optical signal-processing devices, which exploit the nonlinear evolution of the state of polarization of the field that is due to the coupling induced by the Kerr effect between the two orthogonal linearly polarized modes propagating along the fiber. ${ }^{2-5}$

Obviously, central to these investigations is the knowledge of the set of equations describing the evolution of the field propagating inside the fiber in the presence of the nonlinear coupling mechanism induced by the optical Kerr effect. This problem can be attacked either by looking directly for a solution of the field wave equation in the presence of the pertinent nonlinear polarization density ${ }^{6}$ or by relying on coupled-mode theory and letting the role of the coupling perturbation be played by the nonlinear contribution to the refractive index. ${ }^{7}$ Although the formalism developed in Ref. 7 is quite general and capable in principle of dealing with the case of a generic fiber supporting an arbitrary number of copropagating and counterpropagating guided modes, in practice it has been specialized to isotropic waveguides. The influence of birefringence on nonlinear codirectional propagation in a striaght single-mode fiber has indeed been taken into account in a number of papers scattered throughout the literature, ${ }^{4,5,8-10}$ but a general compact expression of the equations of nonlinear evolution, accounting for the si- multaneous presence of the four possible forward- and backward-traveling modes, is not available.

We hope to provide a partial remedy by deriving in this paper the relevant equations for a straight fiber. Once in possession of this information, we address the problem of nonlinear propagation in highly twisted single-mode fibers (spun fibers). The interest in this kind of waveguide arises mostly from the fact that these waveguides have been shown, in the linear regime, to support two copropagating circularly polarized modes, exhibiting negligible mode coupling (a process particularly severe for low- and moderate-birefringence straight fibers and that tends to conceal nonlinear effects) and polarization dispersion. ${ }^{11}$ In Section 3 we derive the set of nonlinear equations obeyed by the four linearly polarized modes copropagating and counterpropagating in an arbitrary twisted fiber. We then introduce the corresponding four circularly polarized states and explicitly show that, in the limit of a high twist rate, mode coupling that is due to fiber imperfections does not play any role on their nonlinear evolution, which turns out to be governed by the same equations as those pertaining to an isotropic medium.

\section{NONLINEAR PROPAGATION IN A STRAIGHT FIBER}

In the presence of imperfections, the electromagnetic field in a waveguide is expressed as the superposition of all the modes of the ideal waveguide. The field propagation is then described in terms of the space-time evolution of the mode amplitudes that obey a coupled set of equations, the coupling coefficients depending on the nature of the waveguide imperfections (coupled-mode theory). This can be done either by starting directly from Maxwell's equation ${ }^{12}$ (which contain first-order derivatives in space and time) or from the wave equation, ${ }^{13}$ the two approaches being equivalent when the radiation modes and the slowly varying field approximation, respectively, are neglected.

The nonlinear part of the refractive index associated with the optical Kerr effect can be considered a perturbation of the linear-waveguide structure, and the evolution of the electromagnetic field can accordingly be investigated by 
means of coupled-mode theory. This has already been done, in the case of linearly polarized light, for a multimode isotropic waveguide (see, for example, Ref. 7), and here we wish to generalize the linear coupled-mode theory to a single-mode birefringent fiber by including the nonlinear-propagation effects.

The transverse part of the analytic signal of the electric field $E$ propagating inside a straight lossless fiber can be written, in term of the guided modes, as

$$
\begin{aligned}
\mathbf{E}(r, z, t)= & \sum_{m} \sum_{\sigma=1}^{2} E_{m}{ }^{\sigma}(r) \hat{\mathbf{e}}_{\sigma}\left\{\exp \left[i \omega_{0} t-i \beta_{m, \sigma}\left(\omega_{0}\right) z\right] \Phi_{m, \sigma}^{+}(z, t)\right. \\
& \left.+\exp \left[i \omega_{0} t+i \beta_{m, \sigma}\left(\omega_{0}\right) z\right] \Phi_{m, \sigma}^{-}(z, t)\right\}
\end{aligned}
$$

where $r=(x, y)$ and $\omega_{0}$ is the midfrequency of the field, $E_{m}{ }^{\sigma}(r)$ is the transverse modal distribution, $\hat{\mathbf{e}}_{1}=\hat{\mathbf{x}}, \hat{\mathbf{c}}_{2}=\hat{\mathbf{y}}$, and the other symbols have the usual meaning (see, for example, Ref. 7). The coupled system of equations describing the evolution of the modal amplitudes $\Phi_{m, \sigma}^{ \pm}$can be obtained as a direct generalization of that pertaining to an isotropic waveguide., ${ }^{71}$ After we recall that, at optical frequencies, the fast-responding electronic processes that are responsible for the nonlinear response of silica give rise to a third-order polarization density of the form ${ }^{15}$

$$
\mathbf{P}^{(3)}=\epsilon_{0} \chi_{3}(\mathbf{E} \cdot \mathbf{E}) E,
$$

the set of nonlinear equations reads as

$$
\begin{aligned}
L_{m, \sigma}^{+} \Phi_{m, \sigma}^{+}= & \sum_{n} \sum_{\sigma^{\prime}} K_{m, n}{ }^{\sigma, \sigma^{\prime}}\left\{\exp \left[i\left(\beta_{m, \sigma}-\beta_{n, \sigma^{\prime}}\right) z\right] \Phi_{n, \sigma^{\prime}}^{+}\right. \\
& \left.+\exp \left[i\left(\beta_{m, \sigma}+\beta_{n, \sigma^{\prime}}\right) z\right] \Phi_{n, \sigma^{\prime}}^{-}\right\}, \\
L_{m, \sigma}^{-} \Phi_{m, \sigma}^{-}= & -\sum_{n} \sum_{\sigma^{\prime}} K_{m, n}{ }^{\sigma, \sigma^{\prime}}\left\{\exp \left[-i\left(\beta_{m, \sigma}+\beta_{n, \sigma^{\prime}}\right) z\right] \Phi_{n, \sigma^{\prime}}^{+}\right. \\
& \left.+\exp \left[-i\left(\beta_{m, \sigma}-\beta_{n, \sigma^{\prime}}\right) z\right] \Phi_{n, \sigma^{\prime}}^{-}\right\},
\end{aligned}
$$

where

$$
K_{m, n}{ }^{\sigma, \sigma^{\prime}}=-i(2 / 3) k n_{2} \int_{-\infty}^{+\infty} \int_{-\infty}^{+\infty} E_{m}{ }^{\sigma}(r) E_{n}{ }^{\sigma^{\prime}}\left(r^{\prime}\right) \hat{\mathbf{e}}_{\sigma} \cdot T \cdot \hat{\mathbf{e}}_{\sigma}^{\prime} \mathrm{d} x \mathrm{~d} y,
$$

$k=\omega_{0} / c, n_{2}=(3 / 8) \chi_{3} / n_{1}\left(n_{1}\right.$ being the linear refractive index), $\mathbf{T}$ is a two-by-two matrix:

$$
\mathbf{T}=\left[\begin{array}{cc}
|E|^{2}+(1 / 2)\left|E_{x}\right|^{2} & (1 / 2) E_{x}{ }^{*} E_{y} \\
(1 / 2) E_{x} E_{y}{ }^{*} & |E|^{2}+(1 / 2)\left|E_{y}\right|^{2}
\end{array}\right],
$$

and $L_{m, \sigma}^{ \pm}$is the differential operator:

$$
\begin{aligned}
& L_{m, \sigma}^{ \pm}=\partial / \partial z \pm\left(1 / V_{m, \sigma}\right) \partial / \partial t \mp\left(i / 2 ! A_{m, \sigma}\right) \partial^{2} / \partial t^{2} \mp \ldots, \\
& V_{m, \sigma}=\left(\mathrm{d} \beta_{m, \sigma} / \mathrm{d} \omega\right)_{\omega=\omega_{0}}^{-1}, \quad A_{m, \sigma}=\left(\mathrm{d}^{2} \beta_{m, \sigma} / \mathrm{d} \omega^{2}\right)_{\omega=\omega_{0}}^{-1},
\end{aligned}
$$

Eqs. (6) and (7) being, respectively, the group velocity and the group-velocity dispersion of the $(m, \sigma)$ mode.

The case of a single-mode fiber can be obtained by setting $m=n=1$, the fiber birefringence in this case being associated with the quantity $\delta \beta=\beta_{1,1}-\beta_{1,2}$. By neglecting on the right-hand sides of Eqs. (3) the fast-oscillating terms containing factors of the kind $\exp (2 i \beta z)$, which is equivalent to the slowly varying approximation, one gets, after considerable algebra, the following set of four nonlinear equations:

$$
\begin{aligned}
L_{1}^{ \pm} \Phi_{1}^{ \pm}= & \mp i R_{11}\left(\left|\Phi_{1}^{ \pm}\right|^{2}+2\left|\Phi_{1}^{\mp}\right|^{2}\right) \Phi_{1}^{ \pm} \mp(2 / 3) i R_{12}\left(\left|\Phi_{2}^{+}\right|^{2}+\left|\Phi_{2}^{-}\right|^{2}\right) \\
& \times \Phi_{1}^{ \pm} \mp(2 / 3) i R_{12} \Phi_{1}^{\mp *} \Phi_{2}^{+} \Phi_{2}^{-} \mp(2 / 3) i R_{12} \\
& \times \exp ( \pm 2 i \delta \beta z) \Phi_{1}^{\mp} \Phi_{2}^{\mp *} \Phi_{2}^{ \pm} \mp(1 / 3) i R_{12} \\
& \times \exp ( \pm 2 i \delta \beta z) \Phi_{1}^{ \pm *} \Phi_{2}^{ \pm 2}, \\
L_{2}^{ \pm} \Phi_{2}^{ \pm}= & \mp i R_{22}\left(\left|\Phi_{2}^{ \pm}\right|^{2}+2\left|\Phi_{2}^{\mp}\right|^{2}\right) \Phi_{2}^{ \pm} \mp(2 / 3) i R_{12}\left(\left|\Phi_{1}^{+}\right|^{2}+\left|\Phi_{1}^{-}\right|^{2}\right) \\
& \times \Phi_{2}^{ \pm} \mp(2 / 3) i R_{12} \Phi_{2}^{\mp *} \Phi_{1}^{+} \Phi_{1}^{-} \mp(2 / 3) i R_{12} \\
& \times \exp (\mp 2 i \delta \beta z) \Phi_{2}^{\mp} \Phi_{1}^{\mp *} \Phi_{1}^{ \pm} \mp(1 / 3) i R_{12} \\
& \times \exp (\mp 2 i \delta \beta z) \Phi_{2}^{ \pm *} \Phi_{1}^{ \pm 2},
\end{aligned}
$$

where the convention has been made to take either the superior or the inferior sign throughout the equations and we have set, for the sake of notational simplicity, $\Phi_{1,1}=\Phi_{1}$, $\Phi_{1,2}=\Phi_{2}, L_{1,1}=L_{1}$, and $L_{1,2}=L_{2}$, and

$$
R_{\sigma, \sigma^{\prime}}=k n_{2} \int_{-\infty}^{+\infty} \int_{-\infty}^{+\infty} E_{1}{ }^{\sigma}(r)^{2} E_{1}{ }^{\sigma^{\prime}}\left(r^{\prime}\right)^{2} \mathrm{~d} x \mathrm{~d} y .
$$

The transverse modal configurations are normalized to one, that is,

$$
\int_{-\infty}^{+\infty} \int_{-\infty}^{+\infty} E_{n}^{\sigma}(r)^{2} \mathrm{~d} x \mathrm{~d} y=1
$$

The set of Eqs. (8) describes the nonlinear evolution of the four copropagating and counterpropagating modes that a single-mode fiber is in general able to support.

The particular case of two copropagating modes is immediately found by putting $\Phi_{1}^{-}=\Phi_{2}^{-}=0$, which yields

$$
\begin{aligned}
L_{1}^{+} \Phi_{1}^{+}= & -i R_{11}\left|\Phi_{1}^{+}\right|{ }^{2} \Phi_{1}^{+}-(2 / 3) i R_{12}\left|\Phi_{2}^{+}\right|{ }^{2} \Phi_{1}^{+}-(1 / 3) i R_{12} \\
& \times \exp (2 i \delta \beta z) \Phi_{1}^{+*} \Phi_{2}^{+2}, \\
L_{2}^{+} \Phi_{2}^{+}= & -i R_{22}\left|\Phi_{2}^{+}\right|{ }^{2} \Phi_{2}^{+}-(2 / 3) i R_{12}\left|\Phi_{1}^{+}\right|{ }^{2} \Phi_{2}^{+}-(1 / 3) i R_{12} \\
& \times \exp (-2 i \delta \beta z) \Phi_{2}^{+*} \Phi_{1}^{+2} .
\end{aligned}
$$

The system of coupled differential equations relevant to four-wave mixing in a waveguide can also be obtained by assuming that $\Phi_{2}^{+}$and $\Phi_{2}^{-}$are the two counterpropagating pump waves and that $\Phi_{1}^{+}$and $\Phi_{1}^{-}$, respectively, are the probe and the phase-conjugate signal. In the limit $\left|\Phi_{2}^{+}\right|,\left|\Phi_{2}^{-}\right| \gg$ $\left|\Phi_{1}^{+}\right|,\left|\Phi_{1}^{-}\right|$(undepleted pump) and for a high-birefringence fiber (such that $1 / \delta \beta$ is small with respect to the length $L_{c}=$ $1 / R_{11}\left|\Phi_{1}\right|\left|\Phi_{2}\right|$ over which a nearly circularly polarized beam significantly rotates the major axis of its polarization ellipse ${ }^{15}$ ), it reads as

$$
\begin{aligned}
& L_{2}^{ \pm} \Phi_{2}^{ \pm}=\mp i R_{22}\left(\left|\Phi_{2}^{ \pm}\right|^{2}+2\left|\Phi_{2}^{\mp}\right|^{2}\right) \Phi_{2}^{ \pm}, \\
& L_{1}^{ \pm} \Phi_{1}^{ \pm}=\mp(2 / 3) i R_{12}\left(\left|\Phi_{2}^{+}\right|^{2}+\left|\Phi_{2}^{-}\right|^{2}\right) \Phi_{1}^{ \pm} \mp(2 / 3) i R_{12} \Phi_{1}^{\mp *} \Phi_{2}^{-} \Phi_{2}^{+} .
\end{aligned}
$$

\section{NONLINEAR PROPAGATION IN A HIGHLY TWISTED FIBER}

Linear propagation inside a single-mode twisted fiber can be described by expanding the field in terms of local normal 
modes. ${ }^{12}$ More precisely, the electric field propagating along the $z$ axis is written as

$$
\begin{aligned}
E(r, z, t)= & E(r) x\left[\exp \left(-i \beta_{1} z+i \omega_{0} t\right) \phi_{1}^{+}(z, t)\right. \\
& \left.+\exp \left(+i \beta_{1} z+i \omega_{0} t\right) \phi_{1}^{-}(z, t)\right]+E(r) y\left[\operatorname { e x p } \left(-i \beta_{2} z\right.\right. \\
& \left.\left.+i \omega_{0} t\right) \phi_{2}^{+}(z, t)+\exp \left(+i \beta_{2} z+i \omega_{0} t\right) \phi_{2}^{-}(z, t)\right],
\end{aligned}
$$

where $x$ and $y$ are the local birefringence axes, $E(r) x$ and $E(r) y$ are the linearly polarized local eigenmodes (that is, in a coordinate system that rotates with the twist), and $\beta_{1}$ and $\beta_{2}$ are the relative propagation constants. The mode amplitudes $\phi_{i}^{ \pm}(i=1,2)$ can then be easily shown, by means of coupled-mode theory, to obey, if chromatic dispersion is neglected, the set of equations

$$
\begin{aligned}
& {\left[\partial / \partial z \pm\left(1 / V_{1}\right) \partial / \partial t\right] \phi_{1}^{ \pm}= \pm(i K+\tau) \exp ( \pm i \delta \beta z) \phi_{2}^{ \pm},} \\
& {\left[\partial / \partial z \pm\left(1 / V_{2}\right) \partial / \partial t\right] \phi_{2}^{ \pm}= \pm(i K-\tau) \exp (\mp i \delta \beta z) \phi_{1}^{ \pm},}
\end{aligned}
$$

where

$$
V_{i}=\mathrm{d} \beta_{i} /\left.\mathrm{d} \omega\right|_{\omega_{0}} ^{-1} \quad(i=1,2)
$$

is the group velocity of the $i$ th mode, $\delta \beta=\beta_{1}\left(\omega_{0}\right)-\beta_{2}\left(\omega_{0}\right)$ is the intrinsic birefringence of the untwisted fiber, $\tau$ is the twist rate, and $K(z)$ is a (real) coupling coefficient resulting from fiber random imperfections.

In the same way, it is possible to show that the set of nonlinear equations describing the nonlinear propagation of the $\phi_{i}^{ \pm}$'s can be obtained simply by adding, on the right-hand sides of Eqs. (14), the same nonlinear terms (NLT's) present on the right-hand sides of Eqs. (8) (provided that one performs the substitution $\left.\Phi_{i}^{ \pm} \rightarrow \phi_{i}^{ \pm}\right)$; thus one gets

$\left[\partial / \partial z \pm 1 /\left(V_{1}\right) \partial / \partial t\right] \phi_{1}^{ \pm}= \pm(i K+\tau) \exp ( \pm i \delta \beta z) \phi_{2}^{ \pm}+\mathrm{NLT}$

[as in Eq. (8a)],

$\left[\partial / \partial z \pm 1 /\left(V_{2}\right) \partial / \partial t\right] \phi_{2}^{ \pm}= \pm(i K-\tau) \exp (\mp i \delta \beta z) \phi_{1}^{ \pm}+\mathrm{NLT}$

[as in Eq. (8b)]. (16b)

It is convenient at this point to introduce the right and left circularly polarized eigenmodes $E(r) e_{r}$ and $E(r) e_{l}$, where

$$
e_{r}=(1 / \sqrt{2})(x+i y), \quad e_{l}=1(\sqrt{2})(x-i y),
$$

and rewrite the electric field in the form

$$
\begin{aligned}
E(r, z, t)= & E(r)\left\{\left[\exp \left(-i \beta_{r} z+i \omega_{0} t\right) \phi_{r}^{+}(z, t)+\exp \left(+i \beta_{l} z\right.\right.\right. \\
& \left.\left.+i \omega_{0} t\right) \phi_{r}^{-}(z, t)\right] e_{r}+\left[\exp \left(-i \beta_{l} z+i \omega_{0} t\right)\right. \\
& \left.\left.\times \phi_{l}^{+}(z, t)+\exp \left(+i \beta_{r} z+i \omega_{0} t\right) \phi_{l}^{-}(z, t)\right] e_{l}\right\},
\end{aligned}
$$

where the propagation constants $\beta_{r}$ and $\beta_{l}$ are $a$ priori unknown. By comparing Eqs. (13) and (18) we can write

$$
\begin{aligned}
& \phi_{1}^{+}=(1 / \sqrt{2}) \exp \left(i \beta_{1} z\right)\left[\exp \left(-i \beta_{r} z\right) \phi_{r}^{+}+\exp \left(-i \beta_{l} z\right) \phi_{l}^{+}\right] \\
& \phi_{2}^{+}=(i / \sqrt{2}) \exp \left(i \beta_{2} z\right)\left[\exp \left(-i \beta_{r} z\right) \phi_{r}^{+}-\exp \left(-i \beta_{l} z\right) \phi_{l}^{+}\right] \\
& \phi_{1}^{-}=(1 / \sqrt{2}) \exp \left(-i \beta_{1} z\right)\left[\exp \left(i \beta_{l} z\right) \phi_{r}^{-}+\exp \left(i \beta_{r} z\right) \phi_{l}^{-}\right] \\
& \phi_{2}^{-}=(i / \sqrt{2}) \exp \left(-i \beta_{2} z\right)\left[\exp \left(i \beta_{l} z\right) \phi_{r}^{-}-\exp \left(i \beta_{r} z\right) \phi_{l}^{-}\right]
\end{aligned}
$$

We now substitute the expression of $\phi_{1}^{+}$and $\phi_{2}^{+}$into the pertinent equations (16a) and (16b). After some tedious if straightforward algebra we obtain

$$
\begin{aligned}
& \left\{-\left(\beta_{1}-\beta_{r}\right)+i \partial / \partial z+\left(i / V_{1}\right) \partial / \partial t\right\} \phi_{r}^{+}+\exp (i \Delta \beta z)\left\{-\left(\beta_{1}-\beta_{l}\right)\right. \\
& \left.\quad+i \partial / \partial z+\left(i / V_{1}\right) \partial / \partial t\right\} \phi_{l}^{+}=-(i K+\tau)\left[\phi_{r}^{+}-\exp (i \Delta \beta z) \phi_{l}^{+}\right] \\
& \quad+(2 / 3) R\left(\left|\phi_{r}^{+}\right|^{2}+2\left|\phi_{l}^{+}\right|^{2}+2\left|\phi_{r}^{-}\right|^{2}+2\left|\phi_{l}^{-}\right|^{2}\right) \phi_{r}^{+} \\
& \quad+(4 / 3) R \phi_{r}^{-} \phi_{l}^{+} \phi_{l}^{-*}+(2 / 3) R \exp (i \Delta \beta z)\left(\left|\phi_{l}^{+}\right|^{2}+2\left|\phi_{r}^{+}\right|^{2}\right. \\
& \left.\quad+2\left|\phi_{r}^{-}\right|^{2}+2\left|\phi_{l}^{-}\right|^{2}\right) \phi_{l}^{+}+(4 / 3) R \exp (i \Delta \beta z) \phi_{r}^{-*} \phi_{r}^{+} \phi_{l}^{-}, \quad(20 \mathrm{a}) \\
& -\exp (i \Delta \beta z)\left\{-\left(\beta_{2}-\beta_{l}\right)+i \partial / \partial z+\left(i / V_{2}\right) \partial / \partial t\right\} \phi_{l}^{+}+\left\{-\left(\beta_{2}-\beta_{r}\right)\right. \\
& \left.\quad+i \partial / \partial z+\left(i / V_{2}\right) \partial / \partial t\right\} \phi_{r}^{+}=(i K-\tau)\left[\exp (i \Delta \beta z) \phi_{l}^{+}+\phi_{r}^{+}\right] \\
& \quad-(2 / 3) R \exp (i \Delta \beta z)\left(\left|\phi_{l}^{+}\right|^{2}+2\left|\phi_{r}^{+}\right|^{2}+2\left|\phi_{r}^{-}\right|^{2}+2\left|\phi_{l}^{-}\right|^{2}\right) \phi_{l}^{+} \\
& \quad-(4 / 3) R \exp (i \Delta \beta z) \phi_{l}^{-} \phi_{r}^{+} \phi_{r}^{-*}+(2 / 3) R\left(\left|\phi_{r}^{+}\right|^{2}+2\left|\phi_{l}^{+}\right|^{2}\right. \\
& \left.\quad+2\left|\phi_{r}^{-}\right|^{2}+2\left|\phi_{l}^{-}\right|^{2}\right) \phi_{r}^{+}+(4 / 3) R \phi_{l}^{-*} \phi_{l}^{+} \phi_{r}^{-},
\end{aligned}
$$

where we have set $\Delta \beta=\beta_{r}-\beta_{l}$ and $R=R_{11}=R_{22}=R_{12}$. If we now assume that $\Delta \beta z \gg 1$, a hypothesis whose validity has to be checked $a$ posteriori since $\Delta \beta$ is still an unknown quantity, we can, thanks to the different scales of spatial variation, equate separately in Eqs. (20a) and (20b) the terms that contain the factor $\exp (i \Delta \beta z)$ and those that do not. This procedure allows us to write two equations for $\phi_{r}^{+}$ and two equations for $\phi_{l}^{+}$. Those for $\phi_{r}^{+}$read as

$$
\begin{aligned}
& {\left[-\left(\beta_{1}-\beta_{r}\right)+i \partial / \partial z+\left(i / V_{1}\right) \partial / \partial t\right] \phi_{r}^{+}=-(i K+\tau) \phi_{r}^{+}} \\
& \quad+(2 / 3) R\left(\left|\phi_{r}^{+}\right|^{2}+2\left|\phi_{l}^{+}\right|^{2}+2\left|\phi_{r}^{-}\right|^{2}+2\left|\phi_{l}^{-}\right|^{2}\right) \phi_{r}^{+} \\
& \quad+(4 / 3) R \phi_{r}^{-} \phi_{l}^{+} \phi_{l}^{-*}, \\
& {\left[-\left(\beta_{2}-\beta_{r}\right)+i \partial / \partial z+\left(i / V_{2}\right) \partial / \partial t\right] \phi_{r}^{+}=(i K-\tau) \phi_{r}^{+}} \\
& \quad+(2 / 3) R\left(\left|\phi_{r}^{+}\right|^{2}+2\left|\phi_{l}^{+}\right|^{2}+2\left|\phi_{r}^{-}\right|^{2}+2\left|\phi_{l}^{-}\right|^{2}\right) \phi_{r}^{+} \\
& \quad+(4 / 3) R \phi_{r}^{-} \phi_{l}^{+} \phi_{l}^{-*},
\end{aligned}
$$

analogous expressions being valid for $\phi_{l}^{+}$. Adding Eq. (21a) to Eq. (21b) yields

$$
\begin{aligned}
& -(1 / 2)\left(\beta_{1}+\beta_{2}-2 \beta_{r}\right) \phi_{r}^{+}+i\left[\partial / \partial z+(1 / 2)\left(1 / V_{1}+1 / V_{2}\right) \partial / \partial t\right] \phi_{r}^{+} \\
& \quad=-\tau \phi_{r}^{+}+(2 / 3) R\left(\left|\phi_{r}^{+}\right|^{2}+2\left|\phi_{l}^{+}\right|^{2}+2\left|\phi_{r}^{-}\right|^{2}+2\left|\phi_{l}^{-}\right|^{2}\right) \phi_{r}^{+} \\
& \quad+(4 / 3) R \phi_{r}^{-} \phi_{l}^{+} \phi_{l}^{-*},
\end{aligned}
$$

which can be satisfied by choosing

$$
\beta_{r}=\left(\beta_{1}+\beta_{2}\right) / 2-\tau \equiv \gamma-\tau
$$

and

$$
\begin{aligned}
{[\partial / \partial z+(1 / V) \partial / \partial t] \phi_{r}^{+}=} & -(2 / 3) i R\left(\left|\phi_{r}^{+}\right|^{2}+2\left|\phi_{l}^{+}\right|^{2}+2\left|\phi_{r}^{-}\right|^{2}\right. \\
& \left.+2\left|\phi_{l}^{-}\right|^{2}\right) \phi_{r}^{+}-(4 / 3) i R \phi_{r}^{-} \phi_{l}^{+} \phi_{l}^{-*}
\end{aligned}
$$

where $1 / V=(1 / 2)\left(1 / V_{1}+1 / V_{2}\right)$. If we had started from the equations for $\phi_{l}^{+}$we would have found that

$$
\beta_{l}=\left(\beta_{1}+\beta_{2}\right) / 2+\tau \equiv \gamma+\tau
$$

and 


$$
\begin{aligned}
{[\partial / \partial z+(1 / V) \partial / \partial t] \phi_{l}^{+}=} & -(2 / 3) i R\left(\left|\phi_{l}^{+}\right|^{2}+2\left|\phi_{r}^{+}\right|^{2}+2\left|\phi_{r}^{-}\right|^{2}\right. \\
& \left.+2\left|\phi_{l}^{-}\right|^{2}\right) \phi_{l}^{+}-(4 / 3) i R \phi_{l}^{-} \phi_{r}^{+} \phi_{r}^{-*} .
\end{aligned}
$$

Equations (24) and (26) show that the amplitudes $\phi_{r}^{+}$and $\phi_{l}^{+}$ of the two circularly polarized states travel with the same group velocity $V$ (no polarization dispersion) and that the fiber imperfections associated with $K(z)$ have no influence on their evolution. Since, according to Eqs. (23) and (25), $\Delta \beta=\beta_{r}-\beta_{l}=-2 \tau$, one has to assume, in order to be consistent with the hypothesis that $\Delta \beta z \gg 1$, a large twist rate $\tau$ (highly twisted fiber). We could now proceed in a similar way and obtain the equations describing the evolution of $\phi_{r}^{-}$and $\phi_{l}^{-}$. We limit ourselves to writing the final equations, which read as

$$
\begin{aligned}
{[\partial / \partial z-(1 / V) \partial / \partial t] \phi_{r}^{-}=} & (2 / 3) i R\left(\left|\phi_{r}^{-}\right|^{2}+2\left|\phi_{l}^{-}\right|^{2}+2\left|\phi_{r}^{+}\right|^{2}\right. \\
& \left.+2\left|\phi_{l}^{+}\right|^{2}\right) \phi_{r}^{-}+(4 / 3) i R \phi_{r}^{+} \phi_{l}^{-} \phi_{l}^{+*},
\end{aligned}
$$

$$
\begin{aligned}
{[\partial / \partial z-(1 / V) \partial / \partial t] \phi_{l}^{-}=} & (2 / 3) i R\left(\left|\phi_{l}^{-}\right|^{2}+2\left|\phi_{r}^{-}\right|^{2}+2\left|\phi_{r}^{+}\right|^{2}\right. \\
& \left.+2\left|\phi_{l}^{+}\right|^{2}\right) \phi_{l}^{-}+(4 / 3) i R \phi_{l}^{+} \phi_{r}^{-} \phi_{r}^{+*} .
\end{aligned}
$$

Equations (23)-(28) completely describe propagation inside a highly twisted optical fiber. In particular, a general solution of the set of Eqs. (24) and (26)-(28) can easily be found ${ }^{16}$ whenever we assume the absence of counterpropagating modes [i.e., $\phi_{r, l}^{+}(z=0, \mathrm{t})=0$ or $\phi_{r, l}^{-}(z=L, t)=0$, where $L$ is the fiber length]. In this case, no power exchange can take place between the two copropagating modes, since $\left|\phi_{r, l}^{+}(z, t)\right|=\left|\phi_{r, l}^{+}(z=0, t-z / V)\right|$ and $\left|\phi_{r, l}^{-}(z, t)\right|=\mid \phi_{r, l}^{-}(z=\mathrm{L}, t+$ $(z-L) / V] \mid$, respectively, so that nonlinear coupling only modifies their phases. This circumstance excludes the possibility of spatial instability in the nonlinear evolution of the state of polarization, in agreement with the results of Ref. 17, where a detailed analysis of forward nonlinear propagation inside a single-mode arbitrary twisted birefringent fiber in the stationary regime has been presented.

Finally, it is useful to write the expression of the field in the laboratory frame, that is, in a reference frame $\left(x^{\prime}, y^{\prime}\right)$ rotating at a rate $\tau$ in the clockwise direction:

$$
\begin{aligned}
& x^{\prime}=\cos (\tau z) x-\sin (\tau z) y, \\
& y^{\prime}=\sin (\tau z) x+\cos (\tau z) y,
\end{aligned}
$$

which reads as

$$
\begin{aligned}
E\left(r^{\prime}, z, t\right)= & E(r)\left\{\left[\exp \left(-i \gamma z+i \omega_{0} t\right) \phi_{r}^{+}(z, t)\right.\right. \\
& \left.+\exp \left(+i \gamma z+i \omega_{0} t\right) \phi_{r}^{-}(z, t)\right] e_{r}{ }^{\prime} \\
& +\left[\exp \left(-i \gamma z+i \omega_{0} t\right) \phi_{l}^{+}(z, t)\right. \\
& \left.\left.+\exp \left(+i \gamma z+i \omega_{0} t\right) \phi_{l}^{-}(z, t)\right] e_{l}^{\prime}\right\},
\end{aligned}
$$

where $e_{r}^{\prime}=\left(x^{\prime}+i y^{\prime}\right) / \sqrt{2}$ and $e_{l}^{\prime}=\left(x^{\prime}+i y^{\prime}\right) / \sqrt{2}$. Thus the four circularly polarized modes of a spun fiber possess, in the limit of a high twist rate, the same propagation constant; since the equations of evolutions of $\phi_{r, l}^{+}$are the same as those for an isotropic medium, ${ }^{18}$ we can conclude that spun fibers also behave as perfectly circular isotropic waveguides from the point of view of nonlinear propagation.

\section{ACKNOWLEDGMENTS}

This research has been supported by the U.S. Office of Naval Research, the National Science Foundation, and NATO research grant $388 / 84$.

* Permanent address, Università dell'Aquila, L'Aquila, Italy. The author is also with the Fondazione Ugo Bordoni, Roma, Italy.

\section{REFERENCES}

1. A. Hasegawa and Y. Kodama, Proc. IEEE 69, 1145 (1981).

2. R. H. Stolen, J. Botineau, and A. Ashkin, Opt. Lett. 7, 512 (1982).

3. K. Kitayama, Y. Kimura, and S. Seikai, Appl. Phys. Lett. 46, 317,623 (1985).

4. H. G. Winful, Appl. Phys. Lett. 47, 213 (1985); Opt. Lett. 11, 33 (1986).

5. B. Daino, G. Gregori, and S. Wabnitz, Opt. Lett. 11, 42 (1986).

6. See, e.g., N. Tzoar and M. Jain, Phys. Rev. A 23, 1266 (1981).

7. B. Crosignani, A. Cutolo, and P. Di Porto, J. Opt. Soc. Am. 72, 1136 (1982).

8. B. Crosignani and P. Di Porto, Opt. Acta 32, 1251 (1985).

9. B. Crosignani, B. Daino, and P. Di Porto, J. Opt. Soc. Am. B 3, 1120 (1986).

10. C. R. Menyuk, IEEE J. Quantum Electron. QE-23, 174 (1987).

11. A. J. Barlow, J. J. Ramskov-Hansen, and D. N. Payne, Appl. Opt. 20, 2962 (1981).

12. D. Marcuse, Theory of Dielectric Optical Waveguides (Academic, New York, 1974).

13. A. Yariv, Optical Electronics (Holt, Rinehart and Winston, New York, 1985)

14. B. Crosignani, in New Directions in Guided Wave and Coherent Optics, D. B. Ostrowsky and E. Spitz, eds. (Nijhoff, The Hague, 1984), Vol. 1, p. 1.

15. P. D. Maker and R. W. Terhune, Phys. Rev. 137, 801 (1965),

16. See, e.g., B. Crosignani and P. Di Porto, J. Opt. Soc. Am. 72, 1553 (1982).

17. F. Matera and S. Wabnitz, Opt. Lett. 11, 467 (1986).

18. A. E. Kaplan and C. T. Law, IEEE J. Quantum Electron. QE-21, 1529 (1985) 


\section{B. Crosignani}

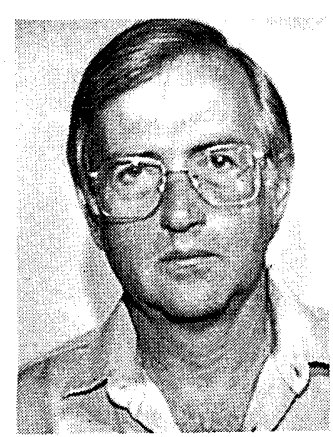

B. Crosignani was born in Rome, Italy, on November 1938. He received the Laurea degree in physics from the University of Rome in 1962 and the Libera Docenza in theoretical physics in 1971. He spent four years as a postdoctoral fellow at the Laboratorio Gas Ionizzati, Frascati, Rome, where he worked on plasma physics. In 1966 he joined Fondazione Ugo Bordoni, Rome, where he conducted research in quantum optics, light scattering, propagation in random media, and linear and nonlinear propagation in optical fibers. In 1971 he joined the Faculty of Electrical Engineering of the University of Rome as adjunct professor of optoelectronics. He joined the Department of Physics, University of Rome, as a professor of optoelectronics. In 1987 he reached the status of full professor of optics in the Department of Physics, University of L'Aquila, L'Aquila, Italy. In 1970-1971 he was a research fellow in the Department of Electrical Engineering, California Institute of Technology, where he has been a visiting associate for part of each year from 1976 to the present. $\mathrm{He}$ is the coauthor of more than 70 papers in scientific journals and two books, Statistical Properties of Scattered Light (Academic, New York, 1975) and Guiding Diffraction and Confinement of Optical Radiation (Academic, New York, 1986). He is also a member of the executive board of the journal Fiber and Integrated Optics. Dr. Crosignani is a member of the Optical Society of America and the Electromagnetic Wave Propagation Panel of AGARD.

\section{A. Yariv}

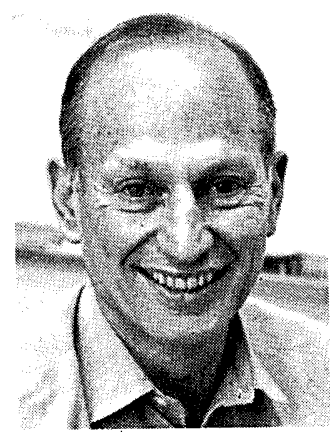

A. Yariv, a native of Israel, obtained the B.S. (1954), M.S. (1956), and Ph.D. (1958) degrees in electrical engineering from the University of California, Berkeley. He went to the Bell Telephone Laboratories, Murray Hill, New Jersey in 1959 , joining the early stages of the laser effort. He went to the California Institute of Technology in 1964 as an associate professor of electrical engineering, becoming a professor in 1966 . In 1980 he became the Thomas G. Myers Professor of Electrical Engineering and Applied Physics. On the technical side, he took part (with various co-workers) in the discovery of a number of early solid-state laser systems, in the formulation of the theory of parametric quantum noise and the prediction of parametric fluorescence, in the invention of the technique of mode-locked ultrashort-pulse lasers and FM lasers, in the introduction of GaAs and CdTe as infrared electrooptic and window materials, in proposing and demonstrating semiconductor-based integrated optics technology, and in pioneering the field of phase-conjugate optics. His present research efforts are in the areas of nonlinear optics, semiconductor lasers, and integrated optics, especially the problem of monolithic integration of transistors, injection lasers, and detectors for high-frequency applications and ultrafast $\left(<10^{-12} \mathrm{sec}\right)$ semiconductor devices and phenomena. Professor Yariv has published some 300 papers in the laser and optics fields and has written a number of basic texts in quantum electronics, optics, and quantum mechanics. He is also an associate editor of Optics Communications and was associate editor of the Journal of Quantum Electronics and the Journal of Applied Physics. He is a member of the American Physical Society, Phi Beta Kappa, the American Academy of Arts and Sciences, and the National Academy of Engineering and is a Fellow of the Institute of Electrical and Electronics Engineers (IEEE) and the Optical Society of America. He has received the 1980 Quantum Electronics Award of the IEEE, the 1985 University of Pennsylvania Pender Award, and the 1986 Optical Society of America Ives Medal. Dr. Yariv is a founder and chairman of the board of Ortel Corporation. 